\title{
0 двух подходах к определению сил притяжения гравитонной модели и их изменений при больших скоростях движения тел
}

\author{
Н.И.Карпенко, НИИСФ РААСН, Москва \\ С.Н.Карпенко, НИИСФ РААСН, Москва
}

Силы тяжести (силы гравитационного, гравитонного притяжения) тел играют важную роль в различных областях науки и техники, в том числе в строительной механике. Так, большинство основных нагрузок, действующих на здания и сооружения, связаны с силами тяжести.

Эти силы определяются на основании закона притяжения тел И. Ньютона. Однако физическая природа переносчика сил притяжения в этом законе остается открытой.

В работах авторов физическая природа формирования и передачи сил тяжести раскрывается на основании установленных закономерностей формирования и реактивного выброса из массы тела потоков гравитонов с последующим рассеиванием и действием рассеянных потоков на встречные массы тел (условно - на встречные тела). Показано, что взаимное действие потоков гравитонов двух тел сводится к закону тяготения И. Ньютона при условии соблюдения третьего закона И. Ньютона: «действие равно противодействию». Однако справедливость действия (дальнодействия) этого закона на расстоянии вызывает вопросы.

В данной статье рассмотрено два подхода гравитонной постановки, при которых закон тяготения И. Ньютона и его третий закон: «действие равно противодействию» - выполняются автоматически.

С учётом особенностей этих подходов рассмотрено развитие гравитонной модели на притяжение тел, движущихся с большими скоростями, когда зависимости гравитонной модели существенно меняются. Усиливается выброс гравитонов, который приводит к сжатию тела и его утяжелению без изменения его начальной массы, а также к другим явлениям.

Ключевые слова: силы тяжести, масса тела, потоки гравитонов, реактивный выброс, скорость тела, время выброса, силы сжатия, деформация тела, область деформации.

On Two Approaches to Determining the Gravitational Forces of the Graviton Model And Its Changes at High Speeds of Motion of Bodies

\section{N.I. Karpenko, NIISF RAACS, Moscow}

S.N. Karpenko, NIISF RAACS, Moscow

The gravity forces (the forces of gravity attraction, gravitational attraction) of bodies play an important role in various fields of science and technology, including building mechanics. Thus, most of the major loads acting on buildings and structures are related to gravity.
These forces are determined on the basis of the law of gravity of I. Newton. However, the physical nature of the carrier of the forces of attraction in this law remains open.

In the works of the authors, the physical nature of the formation and transmission of the gravity forces is revealed on the basis of the established regularities of the formation and reactive ejection of the flows of gravitons from the body mass, followed by scattering and the action of scattered flows on the oncoming masses of bodies (conditionally on the oncoming bodies). It is shown that the mutual action of graviton flows of two bodies reduces to the law of gravity of I. Newton, provided that the third Newton's law "action equals reaction" is observed. However, the validity of the action (long-range) of this law at a distance raises questions.

In this article, we consider two approaches to the graviton formulation, in which Newton's law of gravity and his third law "action equals reaction" are performed automatically.

Taking into account the features of these approaches, we consider the development of the graviton model for bodies moving at high speeds, when the laws of the graviton model change significantly. The release of gravitons increases, which leads to compression of the body and its weighting without changing its initial mass, as well as other effects.

Keywords: gravity, body weight, the flows of gravitons, jet ejection, velocity of the body, time of ejection, the force of contraction, deformation of the body, the region of deformation.

Исходные зависимости гравитонной модели и её особое физическое условие (без учета влияния скорости движения тел, условно в стационарных условиях)

Следуя $[1 ; 2]$, рассмотрим тело шаровидной формы с центром тяжести в центре шара, обозначив: $r_{1}$ - радиус шара, $m_{1}$ - масса шара, $\Delta m_{1}$ - единичная масса (доля массы шара, отнесённая к единице её поверхности)

$$
\Delta \mathrm{m}_{1}=\frac{m_{1}}{4 \pi \mathrm{r}_{1}^{2}}
$$

Массу окружают гравитоны, которые входят в неё и выбрасываются из неё чередующимися всесторонними реактивными потоками со скоростью $V$. В работе [3] рассмотрены иные формирования потоков гравитонов, но все они приводят к одинаковым результатам. Гравитонные потоки вызывают всестороннее сжатие массы и гравитационное 
притяжение к ней других масс. Силу сжатия $f_{1}$ поверхности единичной массы от выброса гравитонов можно определить из известного из теории реактивных двигателей уравнения, представив его в виде:

$$
f_{1}=\frac{\Delta \mathrm{m}_{1}}{\Delta \mathrm{t}_{1}} V=\frac{m_{1} V}{4 \pi r_{1}^{2} \Delta \mathrm{t}_{1}}=\frac{\Delta \bar{\eta}_{1} m_{01} V}{\Delta \mathrm{t}_{1}}=\bar{\eta}_{1} m_{01} V ，
$$

где $\Delta \eta_{1}$ - количество гравитонов, выбрасываемых за время с единицы поверхности шара;

$m_{01}$ - масса, включаемая на выброс одного гравитона (условно единичная доля массы);

$\bar{\eta}_{1}$ - количество гравитонов, выбрасываемых с поверхности шара за единицу времени (условно единичный поток гравитонов):

$$
\bar{\eta}_{1}=\frac{\Delta \eta_{1}}{\Delta \mathrm{t}_{1}} .
$$

Используется связь выражения (2) с известным проявлением гравитации в виде ускорения $a$, с которым притягиваются тела к центру рассматриваемого шара. Согласно, например, [5] гравитационное ускорение на поверхности шара составляет:

$$
a=\frac{\mathrm{\gamma m}_{1}}{r_{1}^{2}},
$$

\section{где $\gamma$ - гравитационная постоянная.}

Ускорение (4) вызывает гравитационное сжатие единичной массы силой $f_{1}$, которая, учитывая (1), (4), будет равна

$$
f_{1}=\Delta \mathrm{m}_{1} \cdot a=\frac{\gamma \mathrm{m}_{1}^{2}}{4 \pi r_{1}^{4}} .
$$

Приравнивая (2) и (5), находим

$$
f_{1}=\frac{\gamma \mathrm{m}_{1}^{2}}{4 \pi r_{1}^{4}}=\frac{\Delta \mathrm{m}_{1}}{\Delta \mathrm{t}_{1}} V=\frac{m_{1} V}{4 \pi r_{1}^{2} \Delta \mathrm{t}_{1}}=\frac{\Delta \eta_{1} m_{01} V}{\Delta \mathrm{t}_{1}}=\eta_{1} m_{01} V .
$$

Единичный поток гравитонов будет равен:

$$
\bar{\eta}_{1}=\frac{\mathrm{\gamma m}_{1}^{2}}{4 \pi r_{1}^{4} m_{01} V} .
$$

Пусть на расстоянии $R$ от тела $m_{1}$ находится тело $m_{2} c$ радиусом $r_{2}$ ( $R$ - расстояние между центрами тяжести $m_{1}$ и $\left.m_{2}\right)$. При этом в зависимостях (1) - (7) формально индекс «1» заменяется на индекс «2». В результате условие (6) и поток гравитонов $\bar{\eta}_{2}$ для тела (2) представляются:

$$
\begin{aligned}
f_{2}=\frac{\gamma \mathrm{m}_{2}^{2}}{4 \pi r_{2}^{4}}=\frac{\Delta \mathrm{m}_{2}}{\Delta \mathrm{t}_{2}} V & =\frac{m_{2} V}{4 \pi r_{2}^{2} \Delta \mathrm{t}_{2}}=\frac{\Delta \eta_{2} m_{02} V}{\Delta \mathrm{t}_{2}}=\bar{\eta}_{2} m_{02} V, \\
\bar{\eta}_{2} & =\frac{\gamma \mathrm{m}_{2}^{2}}{4 \pi r_{2}^{4} m_{02} V} .
\end{aligned}
$$

Рассмотрим два подхода к определению сил действия потоков гравитонов тела $m_{1}$ на тело $m_{2}$ и наоборот (сил взаимодействия).

Подход 1.

Действие потоков $\bar{\eta}_{1}$ на тело $m_{2}$ и наоборот $\bar{\eta}_{2}$ на $m_{1}$ по первому подходу подробно рассмотрено в [2]. При этом учитывается фактор рассеивания потоков на расстоянии $R$, при котором они изменяются до значений:

$$
\bar{\eta}_{12}=\bar{\eta}_{1} r_{1}^{2} / R^{2}, \bar{\eta}_{21}=\bar{\eta}_{2} r_{2}^{2} / R^{2},
$$

и особенности прохождения тел.

В результате установлено, что сила $F_{12}$, с которой потоки гравитонов $\bar{\eta}_{12}$ тела $m_{1}$ действуют на все тело $m_{2^{\prime}}$ и сила обратного направления $F_{21^{\prime}}$ с которой потоки гравитонов $\bar{\eta}_{21}$ тела $m_{2}$ действуют на все тело $m_{1}$, составляют:

$$
\left.\begin{array}{l}
F_{12}=\bar{\eta}_{1} \frac{r_{1}^{2}}{R_{2}} 4 \pi r_{2}^{2} m_{02} V, \\
-F_{2 l}=\bar{\eta}_{2} \frac{r_{2}^{2}}{R^{2}} 4 \pi r_{l}^{2} m_{01} V
\end{array}\right\}
$$

или, учитывая (7), (9)

$$
\left.\begin{array}{l}
F_{12}=\frac{m m_{1}^{2} r_{1}^{2} 4 \pi r_{2}^{2} m_{02} V}{4 \pi r_{1}^{4} m_{01} V R^{2}}=\frac{m m_{1}^{2}\left(4 \pi r_{2}^{2} m_{02}\right)}{\left(4 \pi r_{1}^{2} m_{01}\right) R^{2}}, \\
-F_{21}=\frac{m m_{2}^{2} r_{2}^{2} 4 \pi r_{1}^{2} m_{01} V}{4 \pi r_{2}^{4} m_{02} V R^{2}}=\frac{m m_{2}^{2}\left(4 \pi r_{1}^{2} m_{01}\right)}{\left(4 \pi r_{2}^{2} m_{02}\right) R^{2}} .
\end{array}\right\}
$$

Считаем справедливым физическое условие

$$
\frac{4 \pi r_{1}^{2} m_{01}}{4 \pi r_{2}^{2} m_{02}}=\frac{r_{I}^{2} m_{01}}{r_{2}^{2} m_{02}}=\frac{m_{1}}{m_{2}} .
$$

В результате зависимости (12) приводят к закону тяготения И. Ньютона:

$$
F_{I 2}=-F_{2 l}=\frac{\gamma m_{l} m_{2}}{R^{2}} .
$$

Таким образом, при условии (13) законы «действие равно противодействию» и закон притяжения И. Ньютона выполняются автоматически.

Однако это условие накладывает особенности на значения количества гравитонов, выбрасываемых с единицы поверхности масс. Согласно (6), (8) они будут одинаковыми $\left(\Delta \eta_{1}=\Delta \eta_{2}\right)$, изменяются только величины $m_{01}$ и $m_{02}$, время выброса гравитонов.

Подход 2.

В первом подходе принято, что количество гравитонов, выбрасываемых из масс $m_{1}$ и $m_{2}$, остаётся одинаковым. Это ограничение устраняется, если принять, что единичные доли масс $m_{01}$ и $m_{02}$ в условии (11) должны быть заменены на величины $m_{01}^{\prime}$ и $m_{02}^{\prime}$, при которых все гравитоны потоков будут действовать на встречные тела. При этом условии зависимости (11) представятся в виде:

$$
\left.\begin{array}{l}
F_{12}=\bar{\eta}_{1} \frac{r_{I}^{2}}{R^{2}} 4 \pi r_{2}^{2} m_{02}^{\prime} V, \\
-F_{2 I}=\bar{\eta}_{2} \frac{r_{2}^{2}}{R^{2}} 4 \pi r_{1}^{2} m_{0 I}^{\prime} V .
\end{array}\right\}
$$

или, учитывая (7), (9):

$$
\left.\begin{array}{l}
F_{12}=\frac{m_{1}^{2} r_{1}^{2} 4 \pi r_{2}^{2} m_{02}^{\prime} V}{4 \pi r_{1}^{4} m_{01} V R^{2}}=\frac{m_{1}^{2} r_{2}^{2} m_{02}^{\prime}}{r_{1}^{2} m_{01} R^{2}} \\
-F_{21}=\frac{m m_{2}^{2} r_{2}^{2} 4 \pi r_{1}^{2} m_{01}^{\prime} V}{4 \pi r_{2}^{4} m_{02} V R^{2}}=\frac{m m_{2}^{2} r_{1}^{2} m_{01}^{\prime}}{r_{2}^{2} m_{02} R^{2}} .
\end{array}\right\}
$$


Из зависимостей (6), (8) и при принятых величинах $m_{01}^{\prime}$ и $m_{02}^{\prime}$ следует:

$$
\left.\begin{array}{c}
\frac{m_{1}}{4 \pi r_{I}^{2}}=\Delta \eta_{1} m_{01}, \frac{m_{2}}{4 \pi r_{2}^{2}}=\Delta \eta_{1} m_{02}^{\prime}, \\
\frac{m_{2}}{4 \pi r_{2}^{2}}=\Delta \eta_{2} m_{02}, \frac{m_{1}}{4 \pi r_{1}^{2}}=\Delta \eta_{2} m_{01}^{\prime},
\end{array}\right\},
$$

в результате:

$$
\begin{aligned}
\frac{m_{2}}{m_{1}} & =\frac{4 \pi r_{2}^{2} \Delta \eta_{I} m_{02}^{\prime}}{4 \pi r_{1}^{2} \Delta \eta_{1} m_{01}}=\frac{r_{2}^{2} m_{02}^{\prime}}{r_{I}^{2} m_{01}}, \\
\frac{m_{1}}{m_{2}} & =\frac{r_{1}^{2} m_{01}^{\prime}}{r_{12}^{2} m_{02}} .
\end{aligned}
$$

Зависимости (15) с учётом (18) приводят к условиям:

$$
F_{12}=\frac{\gamma m_{1} m_{2}}{R^{2}}, \quad-F_{2 l}=\frac{\gamma m_{1} m_{2}}{R^{2}} .
$$

Таким образом, при условиях (18) закон «действие равно противодействию» и закон притяжения И. Ньютона выполняются автоматически.

Однако на значения $m_{01}^{\prime}$ и $m_{02}^{\prime}$ могут накладываться ограничения. При выходе значений $m_{01}^{\prime}$ и $m_{02}^{\prime}$ за ограничения закон притяжения И. Ньютона будет нарушаться.

\section{Уравнения гравитонной модели с учётом влияния} скорости движения тел

Следуя [3; 4] рассматриваются тела $m_{1}$ и $m_{2}$ (сокращенно $\left.m_{\mathrm{i}}\right)$, которые переходят к движению соответственно со скоростями $v_{i}$ относительно своего начального состояния, соответствующего $v_{\mathrm{i}}=0$ (здесь и ниже $i=1,2$ ). Полагаем, что при этом сила $f_{\mathrm{i}}$ увеличивается до значений $\tilde{f}_{\mathrm{i}}$, приводя к деформированию тела. В результате радиус тел уменьшается до значений $\tilde{r}_{\mathrm{i}}$, приводя к изменению единичных масс $\Delta m_{1}$ до значений:

$$
\Delta \widetilde{m}_{i}=\frac{m_{i}}{4 \pi \widetilde{r}_{i}^{2}}=\Delta m_{i} \frac{r_{i}^{2}}{\widetilde{r}_{i}^{2}} .
$$

При этом полагаем, что массы $m_{\mathrm{i}}$ не изменяются. Примем закономерность изменения радиуса в виде:

$$
\widetilde{r}_{i}=r_{i} \sqrt{1-\frac{v_{i}^{2}}{n_{i}^{2} V^{2}}}=r_{i} \beta_{i}, \quad \beta_{i}=\frac{\widetilde{r}_{i}}{r_{i}}, i=1,2,
$$

где $\beta_{\mathrm{i}}$ и $n_{\mathrm{i}}$ - обобщённые параметры податливости тел сжатию, зависящие от их физико-механических характеристик, с параметром $n_{\mathrm{i}}$ связан ещё фактор торможения гравитационным полем, в котором тела движутся.

С учетом (21)

$$
\Delta \widetilde{m}_{i}=\Delta m_{i} / \beta_{i}^{2} .
$$

Полагаем, что при этом могут изменяться единичные доли масс $m_{0 \mathrm{i}}$ и время выброса гравитонов $\Delta t_{1}$ до значений:

$$
\tilde{m}_{0 \mathrm{i}}=m_{0 \mathrm{i}} \gamma_{0 \mathrm{i}}, \quad \Delta \tilde{t}_{i}=\Delta \mathrm{t}_{i} \gamma_{\mathrm{ti}},
$$

где $\gamma_{0 \mathrm{i}}$ и $\gamma_{\mathrm{ti}},(i=1,2$,$) - коэффициенты изменения.$

Гравитонные уравнения (2), (8) для движущихся тел представляются в виде

$$
\widetilde{f}_{i}=\frac{\Delta \widetilde{m}_{i}}{\Delta \widetilde{t}_{i}} V=\frac{\Delta \widetilde{\eta}_{i} \widetilde{m}_{0 i} V}{\Delta \widetilde{t}_{i}}=\widetilde{\eta}_{i} \widetilde{m}_{0 i} V,
$$

или, учитывая (22), (2) и (8), а также (23):

$\tilde{f}_{i}=\frac{\Delta m_{i} V}{\Delta \widetilde{t}_{i} \beta_{i}^{2}}=\frac{\Delta \eta_{i} m_{0 \mathrm{i}} V}{\Delta \widetilde{t}_{i} \beta_{i}^{2}}=\frac{\Delta \eta_{i} m_{0 \mathrm{i}} V}{\Delta \mathrm{t}_{i} \gamma_{\mathrm{ti}} \beta_{i}^{2}}=\bar{\eta}_{i} \frac{m_{0 \mathrm{i}} V}{\gamma_{\mathrm{ti}} \beta_{i}^{2}}=\bar{\eta}_{i} \frac{\widetilde{m}_{0 \mathrm{i}} V}{\gamma_{\mathrm{ti}} \gamma_{0 \mathrm{i}} \beta_{i}^{2}}=\widetilde{\eta}_{i} \widetilde{m}_{0 \mathrm{i}} V$.

Из (25) следует

$$
\tilde{\eta}_{i}=\bar{\eta}_{i} \frac{1}{\gamma_{t i} \gamma_{0 i} \beta_{i}^{2}}=\bar{\eta}_{i} \varphi_{i}
$$

где $\varphi_{\mathrm{i}}$ - коэффициент усиления единичных потоков гравитонов:

$$
\varphi_{i}=\frac{1}{\gamma_{t i} \gamma_{0 i} \beta_{i}^{2}} .
$$

Подстановка значений $\bar{\eta}_{\mathrm{i}}$, определяемых по формулам (7) и (9), в (26) приводит к зависимости:

$$
\tilde{\eta}_{i}=\frac{\gamma m_{i}^{2} \varphi_{i}}{4 \pi r_{i}^{4} m_{0 i} V}=\frac{\gamma m_{i}^{2}}{4 \pi r_{i}^{4} m_{0 i} V \gamma_{t i} \gamma_{0 i} \beta_{i}^{2}} .
$$

Из (28) следует, что усиление потоков гравитонов тел, движущихся с большими скоростями, равносильно увеличению массы тела $m_{\mathrm{i}}$ до значений:

$$
m_{i} \sqrt{\varphi_{i}}=m_{i} \frac{1}{\beta_{i} \sqrt{\gamma_{t i} \gamma_{0 i}}} .
$$

В случае, если единичные доли масс $m_{0 \mathrm{i}}$ тела и время выброса гравитонов $\Delta \tilde{t_{\mathrm{i}}}$ не изменяются, то есть при

$$
\tilde{m}_{0 \mathrm{i}}=m_{0 \mathrm{i}}, \Delta \tilde{t}_{\mathrm{i}}=\Delta t_{\mathrm{i}},
$$

усиление выброса потоков гравитонов равносильно увеличению массы $m_{\mathrm{i}}$ до значений $m_{\mathrm{i}} / \beta_{\mathrm{i}}$.

При $V=c$ (эта возможность обсуждалась в [3; 4]), где c - скорость света, это увеличение совпадает с полученным увеличением массы в опытах Лоренца. Формула Лоренца также используется в специальной теории относительности А. Эйнштейна в иной трактовке. В представленной гравитонной модели такой эффект достигается без изменения массы тела.

Для дальнейших построений значение $r_{\mathrm{i}}^{2}$ в зависимости (28) выразим на основании (21) через значение $\tilde{r}_{i}$, в результате:

$$
\tilde{\eta}_{i}=\frac{\gamma m_{i}^{2}}{4 \pi r_{l}^{2} m_{0 i} \widetilde{r}_{i}^{2} \gamma_{t i} \gamma_{0 i} V} \text {. }
$$

Соответственно для первого тела $(i=1)$ и второго тела $(i=2)$ потоки $\tilde{\eta}_{\mathrm{i}}$ будут равны:

$$
\begin{aligned}
& \widetilde{\eta}_{i}=\frac{\gamma m_{1}^{2}}{4 \pi r_{1}^{2} m_{01} \widetilde{r}_{1}^{2} \gamma_{t 1} \gamma_{01} V}, \\
& \widetilde{\eta}_{2}=\frac{\gamma m_{2}^{2}}{4 \pi r_{2}^{2} m_{02} \widetilde{r}_{2}^{2} \gamma_{t 2} \gamma_{02} V} .
\end{aligned}
$$

Рассмотрение сил взаимного притяжения тел при больших скоростях их движения с позиции второго подхода

При достижении потоками $\bar{\eta}_{1}$ тела $m_{2}$ и потоками $\bar{\eta}_{2}$ тела $m_{1}$ потоки будут изменяться. Обозначим изменяемые потоки 
соответственно $\bar{\eta}_{12}$ и $\bar{\eta}_{21}$. При этом, согласно [3], на изменение потоков будут влиять два фактора: фактор рассеивания на расстояние $R$ и фактор локального усиления внутри пространства $\left(r_{\mathrm{i}}-\tilde{r}_{\mathrm{i}}\right)$, который пока представим параметрами $\lambda_{1}$ и $\lambda_{2}$. С учётом этих факторов потоки $\tilde{\eta}_{12}$ и $\tilde{\eta}_{21}$ составят:

$$
\begin{aligned}
& \tilde{\eta}_{12}=\tilde{\eta}_{1} \frac{r_{1}^{2} \lambda_{2}}{R^{2}}, \\
& \tilde{\eta}_{21}=\tilde{\eta}_{2} \frac{r_{2}^{2} \lambda_{1}}{R^{2}} .
\end{aligned}
$$

Общие потоки, которые обозначим $\tilde{\eta}_{\mathrm{s} 12}, \tilde{\eta}_{\mathrm{s} 21}$, пересекающие полностью поверхности $m_{2}$ и $m_{1}$, согласно [1; 2] будут равны:

$$
\widetilde{\eta}_{s l 2}=\widetilde{\eta}_{12} \cdot 4 \pi \widetilde{r}_{2}^{2}, \widetilde{\eta}_{s 2 l}=\widetilde{\eta}_{21} \cdot 4 \pi \widetilde{r}_{1}^{2} .
$$

Во втором подходе сила $\tilde{F}_{12}$ с которой $m_{1}$ действует на $m_{2}$, и сила $\tilde{F}_{21}$, с которой, наоборот, $m_{2}$ действует на $m_{1}$, будут равны:

$$
\widetilde{F}_{12}=\widetilde{\eta}_{s 12} \widetilde{m}_{02}^{\prime} V,-\widetilde{F}_{2 I}=\widetilde{\eta}_{s 21} \widetilde{m}_{01}^{\prime} V
$$

где $\tilde{m}_{01}^{\prime} \neq \tilde{m}_{01}, \tilde{m}_{02}^{\prime} \neq \tilde{m}_{02}$.

Учитывая (30) - (33), силы притяжения $\tilde{F}_{12}$ и $\tilde{F}_{21}$, можно представить в виде

$$
\begin{aligned}
& \widetilde{F}_{12}=\frac{\gamma m_{1}^{2} \lambda_{2} 4 \pi \widetilde{r}_{2}^{2} \widetilde{m}_{02}^{\prime}}{4 \pi r_{1}^{2} m_{01} \gamma_{\mathrm{t} 1} \gamma_{01} R^{2}}, \\
& -\widetilde{F}_{21}=\frac{\gamma m_{2}^{2} \lambda_{1} 4 \pi \widetilde{r}_{1}^{2} \widetilde{m}_{01}^{\prime}}{4 \pi r_{2}^{2} m_{02} \gamma_{\mathrm{t} 2} \gamma_{02} R^{2}} .
\end{aligned}
$$

Представим по аналогии (23)

$$
\widetilde{m}_{0 i}^{\prime}=\gamma_{0 i}^{\prime} m_{0 i}^{\prime}, i=1,2,
$$

где $\gamma_{0 \mathrm{i}}^{\prime}$ - коэффициенты изменения единичных масс $m_{0 \mathrm{i}}^{\prime}$.

Выражая в (34) $\tilde{r}_{\mathrm{i}}^{2}$ через $r_{\mathrm{i}}^{2}$ и $\tilde{m}_{0 \mathrm{i}}^{\prime}$ через $m_{0 \mathrm{i}}^{\prime}$ на основании (16), (7), (35) и учитывая закономерность (18), приходим к зависимостям:

$$
\begin{aligned}
& \widetilde{F}_{12}=\frac{\gamma m_{1}^{2} \lambda_{2}\left(4 \pi r_{2}^{2} m_{02}^{\prime}\right) \beta_{2}^{2} \gamma_{02}^{\prime}}{\left(4 \pi r_{1}^{2} m_{01}\right) \gamma_{11} \gamma_{01} R^{2}}=\frac{\gamma m_{1} m_{2} c_{12}}{R^{2}}, \\
& -\widetilde{F}_{2 I}=\frac{\gamma m_{2}^{2} \lambda_{1}\left(4 \pi r_{I}^{2} m_{01}^{\prime}\right) \beta_{I}^{2} \gamma_{01}^{\prime}}{\left(4 \pi r_{2}^{2} m_{02}\right) \gamma_{12} \gamma_{02} R^{2}}=\frac{\gamma m_{1} m_{2} c_{21}}{R^{2}},
\end{aligned}
$$

где $C_{12}, C_{21}$ - коэффициенты нарушения закона тяготения И. Ньютона при больших скоростях движения тел:

$$
\begin{aligned}
& C_{12}=\frac{\beta_{2}^{2} \lambda_{2} \gamma_{02}^{\prime}}{\gamma_{11} \gamma_{01}}, \\
& C_{21}=\frac{\beta_{1}^{2} \lambda_{1} \gamma_{01}^{\prime}}{\gamma_{12} \gamma_{02}} .
\end{aligned}
$$

В случае, если локальное усиление, как принято в [3], представляется зависимостями (27):

$$
\lambda_{1}=\varphi_{1}=\frac{1}{\gamma_{t 1} \gamma_{01} \beta_{1}^{2}}, \quad \lambda_{2}=\varphi_{2}=\frac{1}{\gamma_{t 2} \gamma_{02} \beta_{2}^{2}}
$$

зависимости (37) принимают вид:

$$
C_{I 2}=\frac{\gamma_{02}^{\prime}}{\gamma_{t 1} \gamma_{01} \gamma_{t 2} \gamma_{02}}, C_{2 l}=\frac{\gamma_{01}^{\prime}}{\gamma_{t 2} \gamma_{02} \gamma_{t 1} \gamma_{01}} .
$$

В случае, если локальное усиление отсутствует (при $\varphi_{1}$ $=\varphi_{2}=1$ ),

$$
C_{12}=\frac{\beta_{2}^{2} \gamma_{02}^{\prime}}{\gamma_{t 1} \gamma_{01}}, C_{21}=\frac{\beta_{1}^{2} \gamma_{01}^{\prime}}{\gamma_{t 2} \gamma_{02}} .
$$

К условиям (36), (37) сводится много вариантов значений (36) - (40). Рассмотрим для примера три варианта из них (1.1, 1.2, 1.3).

1.1. Время на выброс гравитонов $\Delta \tilde{t}_{\mathrm{i}}$ и значения $\tilde{m}_{0 \mathrm{i}}, \tilde{m}_{0 \mathrm{i}}^{\prime}$ не меняются $\left(\Delta \tilde{t}_{\mathrm{i}}=\Delta t_{\mathrm{i}}, \tilde{m}_{0 \mathrm{i}}=m_{0 \mathrm{i}}, \tilde{m}_{0 \mathrm{i}}^{\prime}=m_{0 \mathrm{i}}^{\prime}\right)$. При этом согласно (23), (35):

$$
\gamma_{\mathrm{t} 1}=\gamma_{\mathrm{t} 2}=1, \gamma_{01}=\gamma_{02}=1, \gamma_{01}^{\prime}=\gamma_{02}^{\prime}=1 \text {. }
$$

В случае соблюдения этих условий из зависимостей (38), (39) следует:

$$
C_{12}=C_{21}=1 \text {, }
$$

и закон И. Ньютона согласно (36) будет соблюдаться:

$$
\begin{aligned}
& \widetilde{F}_{12}=\frac{\gamma m_{1} m_{2} c_{12}}{R^{2}}=\frac{\gamma m_{1} m_{2}}{R^{2}}, \\
& -\widetilde{F}_{2 l}=\frac{\gamma m_{1} m_{2} c_{2 l}}{R^{2}}=\frac{\gamma m_{1} m_{2}}{R^{2}} .
\end{aligned}
$$

Если соблюдаются условия (40), притяжения между телами уменьшаются, и закон равенства взаимного тяготения нарушается:

$$
\begin{gathered}
\widetilde{F}_{12}=\frac{\gamma m_{1} m_{2} \beta_{2}^{2}}{R^{2}}, \\
-\widetilde{F}_{2 l}=\frac{\gamma m_{1} m_{2} \beta_{I}^{2}}{R^{2}} .
\end{gathered}
$$

1.2. Время на выброс гравитонов удлиняется, а значения $m_{0 \mathrm{i}}$ не изменяются:

$$
\Delta \widetilde{t}_{i}=\Delta t_{i} \frac{r_{i}}{\widetilde{r}_{i}}=\frac{\Delta t_{i}}{\beta_{i}}, \quad \widetilde{m}_{0 i}=m_{0 i}, \quad \widetilde{m}_{0 i}^{\prime}=m_{0 i}^{\prime}
$$

при этом:

$$
\gamma_{t i}=\frac{1}{\beta_{i}}, \quad \gamma_{0 i}=\gamma_{0 i}^{\prime}=1 .
$$

В случае соблюдения условия (39) :

$$
C_{12}=C_{21}=\beta_{1} \cdot \beta_{2}
$$

и закон равенства сил взаимного тяготения соблюдается в форме:

$$
\widetilde{F}_{12}=-\widetilde{F}_{2 I}=\frac{\gamma m_{1} m_{2} \beta_{1} \beta_{2}}{R^{2}} .
$$

В случае соблюдения условий (40):

$$
C_{12}=\beta_{2}^{2} \cdot \beta_{1}, C_{21}=\beta_{1}^{2} \cdot \beta_{2},
$$


закон равенства сил взаимного тяготения нарушается:

$$
\widetilde{F}_{12}=\frac{\gamma m_{1} m_{2} \beta_{1} \beta_{2}^{2}}{R^{2}}, \quad-\widetilde{F}_{2 I}=\frac{\gamma m_{1} m_{2} \beta_{1}^{2} \beta_{2}}{R^{2}} .
$$

При этом силы взаимного тяготения в обеих случаях значительно уменьшаются.

1.3. Время на выброс гравитонов укорачивается, а значения $\tilde{m}_{0 \mathrm{i}}, \tilde{m}_{0 \mathrm{i}}^{\prime}$ не изменяются:

$$
\Delta \tilde{t}_{i}=\Delta t_{i} \frac{\tilde{r}_{i}}{r_{2}}=\Delta t_{i} \beta_{i}, \quad \tilde{m}_{0 i}=m_{0 I}, \quad \tilde{m}_{0 i}^{\prime}=m_{0 I}^{\prime}
$$

и соответственно:

$$
\gamma_{t i}=\beta_{i}, \quad \gamma_{0 i}=\gamma_{0 i}^{\prime}=1
$$

При этом, в случае соблюдения условий (39):

$$
C_{12}=C_{21}=\frac{1}{\beta_{1} \cdot \beta_{2}}
$$

и силы взаимного притяжения согласно (36) увеличиваются при соблюдении их равенства

$$
\widetilde{F}_{12}=-\widetilde{F}_{21}=\frac{\gamma m_{1} m_{2}}{\beta_{1} \beta_{2} R^{2}} .
$$

При соблюдении условия (40):

$$
C_{12}=\beta_{2}^{2} / \beta_{1}, C_{2 l}=\beta_{1}^{2} / \beta_{2},
$$

и силы тяготения составляют:

$$
\widetilde{F}_{12}=\frac{\gamma m_{1} m_{2} \beta_{2}^{2}}{R^{2} \beta_{1}}, \quad-\widetilde{F}_{21}=\frac{\gamma m_{1} m_{2} \beta_{1}^{2}}{R^{2} \beta_{2}} .
$$

Согласно (48), например, при $\beta_{2} \rightarrow 1$, а $\beta_{1} \rightarrow 0$, сила притяжения $\tilde{F}_{12}$ телом $m_{1}$ тела $m_{2}$ будет стремиться к бесконечности, в то время как сила притяжения ${\underset{F}{21}}_{21}$ телом $m_{2}$ тела $m_{1}$ будет стремиться к нулю.

$$
\star * *
$$

Рассмотрены основные зависимости гравитонной модели притяжения тел и установлены закономерности, при которых закон притяжения тел И. Ньютона и его третий закон - «действие равно противодействию» - на расстоянии без взаимного контакта тел соблюдаются автоматически. Рассмотрено развитие гравитонной модели притяжения тел, движущихся с большими скоростями, с учётом двух подходов. При этом закономерности гравитонной модели претерпевают существенные изменения: усиливается выброс гравитонов, укорачивается или удлиняется время выброса гравитонов, происходит сжатие тела и его утяжеление или облегчение без изменения начальной массы.

На основании рассмотренных подходов дана количественная оценка указанным факторам, которые могут значительно усиливать, ослаблять или в отдельных случаях выравнивать силы притяжения одного тела к другому, при нарушении или соблюдении закона равенства сил притяжения виде закона И. Ньютона или в иной форме. Показано, что при определенных параметрах гравитонной модели для движущихся с большими скоростями тел полученное их утяжеление при неизменной массе согласуется с данными ответов Лоренца.

Рассмотрены также условия, при которых одно тело будет притягивать окружающие его тела с силой, стремящейся к бесконечности, в то время как силы притяжения этого тела окружающими его телами будут стремиться к нулю.

\section{Лuтература}

1. Карпенко Н.И. 0 реактивной природе сил тяжести / Н.И. Карпенко, С.Н. Карпенко // Academia. Архитектура и строительство. - 2014. - № 1. - С. 87-88.

2. Карпенко Н.И. 0 физической природе формирования и передачи сил тяжести / Н.И. Карпенко, С.Н. Карпенко // Естественные и технические науки. - 2015. - № 4 (82). - С. 26-31.

3. Карпенко Н.И. К определению сил тяжести тел при больших скоростях их движения / Н.И. Карпенко, С.Н. Карпенко // Academia. Архитектура и строительство. - 2017. - № 4. - C. 103-106.

4. Карпенко Н.И. 0 физических предпосылках и построении гравитационной (гравитонной) модели притяжения тел при больших скоростях их движения / Н.И. Карпенко, С.Н. Карпенко // Естественные и технические науки. - 2017. - № 11 (113). - С. 224-231.

5. Кемпфер Ф.А. Путь в современную физику / Ф.А. Кемпфер; перевод с английского. - М. : Мир, 1972.

\section{References}

1. Karpenko N.I., Karpenko S.N. 0 reaktivnoi prirode sil tyazhesti [On reaction nature of gravity]. In: Academia. Arkhitektura i stroitel'stvo [Academia. Architecture and Construction], 2014, no. 1, pp. 87-88. (In Russ., abstr. in Engl.)

2. Karpenko N.I., Karpenko S.N. 0 fizicheskoi prirode formirovaniya i peredachi sil tyazhesti [On physical Nature of Generating and Transmitting Gravity Forces]. In: Estestvennye i tekhnicheskie nauki [Natural and Technical Sciences], 2015, no. 4 (82), pp. 26-31. (In Russ., abstr. in Engl.)

3. Karpenko N.I., Karpenko S.N. Kopredeleniyu sil tyazhesti tel pri bol'shikh skorostyakh ikh dvizheniya [To the Definition of gravity force of Bodies at High Speeds]. In: Academia. Arkhitektura i stroitel'stvo [Academia. Architecture and Construction], 2017, no. 4, pp. 103-106. (In Russ., abstr. in Engl.)

4. Karpenko N.I., Karpenko S.N. 0 fizicheskikh predposylkakh $\mathrm{i}$ postroenii gravitatsionnoi (gravitonnoi) modeli prityazheniya tel pri bol'shikh skorostyakh ikh dvizheniya [About the Physical Premises and Building Gravity (Graviton) Model of Attraction of Bodies at High Speeds]. In: Estestvennyei tekhnicheskie nauki [Natural and Technical Sciences], 2017, 11 (113), pp. 224-231. (In Russ., abstr. in Engl.)

5. Kempfer F.A. Put' $v$ sovremennuyu fiziku [The way to modern physics] / F.A. Kempfer; translated from English. Moscow, Mir Publ., 1972. 
Карпенко Николай Иванович (Москва). Доктор технических наук, профессор, академик РААСН. Академик-секретарь РААСН, главный научный сотрудник лаборатории «Проблемы прочности и качества в строительстве» ФГБУ «Научно-исследовательский институт строительной физики Российской академии архитектуры и строительных наук (127238, Москва, Локомотивный проезд, 21. НИИСФ РААСН). Эл. почта: niisf_lab9@mail.ru.

Карпенко Сергей Николаевич (Москва). Доктор технических наук. Главный научный сотрудник лаборатории «Проблемы прочности и качества в строительстве» ФГБУ «Научно-исследовательский институт строительной физики Российской академии архитектуры и строительных наук (127238, Москва, Локомотивный проезд, 21. НИИСФ PAACH). E-mail: niisf_lab9@mail.ru.

Karpenko Nikolai I. (Mosccow). Doctor of technical sciences, professor, academician of RAACS. Academician secretary of the RAACS, chief research officer of the laboratory "Problems of Strength and Quality in Construction" in the Research Institute of Building Physics of RAACS (21 Lokomotivny proezd, Moscow, 127238. NIISF RAACS). E-mail: niisf_lab9@mail.ru.

Karpenko Sergey N. (Moscow). Doctor of technical sciences. Chief researcher of thelaboratory "Problems of Strength and Quality in Construction" in the Research Institute of Building Physics of RAACS (21 Lokomotivny proezd, Moscow, 127238. NIISF RAACS). E-mail: niisf_lab9@mail.ru. 